\title{
NONLINEAR INELASTIC LIGHT SCATTERING IN A SMECTIC LIQUID CRYSTAL
}

\author{
B. Pura, W. Jęda and A. Zagórski \\ Institute of Physics, Warsaw University of Technology \\ Koszykowa 75, 00-662 Warszawa, Poland
}

(Received July 31, 1997; revised version November 8, 1997)

Results of measurements of the intensity of light scattered by a smectic liquid crystal are reported for three phases of the specimen. A strong dependence of the scattered intensity on the relative directions of polarization vector and molecular orientation is observed. A mechanism of light interaction with material is proposed, it is attributed to Brillouin - like scattering of light on director - system excitations.

PACS numbers: $42.70 . \mathrm{Df}, 42.65 .-\mathrm{k}$

\section{Introduction}

Liquid crystals belong to the intensively studied materials in modern optics. Their interaction with strong laser light has a highly nonlinear character, leading to much stronger effects than in other (inorganic) materials [1, 2]. The majority of papers on this subject deals with nematic liquid crystals, whose symmetry is lower. Their optical properties were described in a number of papers (see, for instance, Refs. [3, 4]).

Smectic liquid crystals (SLC) have a more ordered molecular structure than nematics, which modifies their interaction with light. Knowledge of the microscopic mechanisms responsible for this interaction is, however, very unsatisfactory. The aim of this work was to measure the dependence of the scattered light intensity on the polarization state of the incoming beam for a representative of a class of smectic liquid crystals.

We used a SLC sample consisting of the compound: ethyl 4-(4'-octyloxybenzoiloxy)-benzoate. This material can exist in a few phases. Below the temperature $t_{c}=35.5^{\circ} \mathrm{C}$ it has a solid, crystalline structure. Above this temperature it acquires the smectic $C\left(S_{c}\right)$ structure, which for $t_{a}=42.2^{\circ} \mathrm{C}$ undergoes a second phase transition to the smectic $A\left(S_{a}\right)$ form. The next transition (to an isotropic liquid state $I$ ) occurs at $t_{I}=57.2^{\circ} \mathrm{C}$. 


\section{Experiment}

The LC sample was initially ordered in the smectic $C$ phase by gradual cooling from $80^{\circ} \mathrm{C}$ to $40^{\circ} \mathrm{C}$ in the magnetic field of the intensity $B$ equal to $0.5 \mathrm{~T}$. The degree of order was then checked by means of X-ray diffraction. The intensity of the respective Bragg scattering was 200 times larger than for the disordered sample. The direction of the magnetic field was chosen in such a way that on the average the long molecular axes were parallel to the surface of the sample.

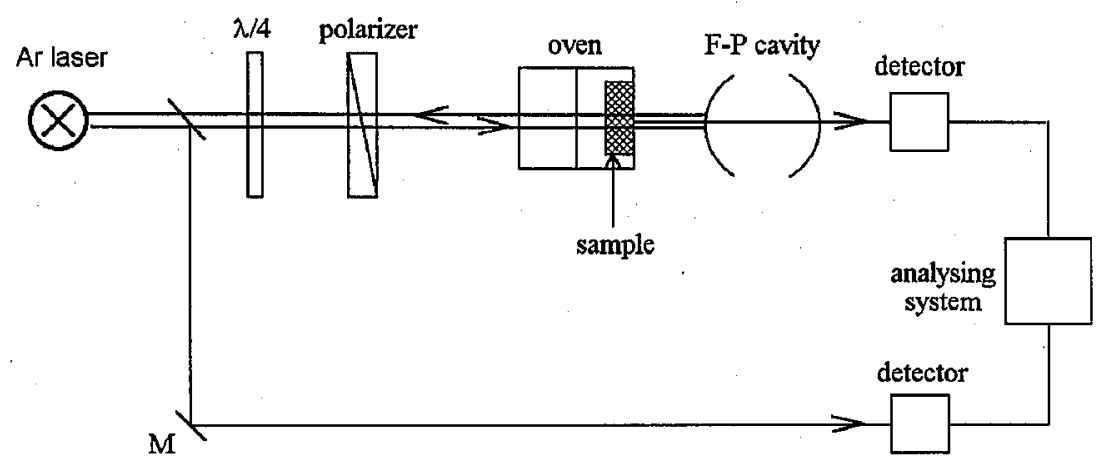

Fig. 1. Experimental setup.

The thus ordered SLC-film was placed in the experimental setup shown in Fig. 1. We used the argon laser (LEXEL 3500) which operates at $\lambda=514 \mathrm{~nm}$. The laser beam was polarized by means of a polarizer. The SLC was enclosed in a quartz oven having the form of a ring with the diameter of $4 \mathrm{~mm}$. The thickness of the SLC-film was about $30 \mu \mathrm{m}$. The sample could be rotated around the axis perpendicular to its surface, which coincided with the laser beam. In this way we could change the angle between the molecular director $n$ and the electric field $\boldsymbol{E}$ of the incident (polarized) beam.

The measurements were subsequently repeated for three phases: $S_{a}, S_{c}$ and $I$. For each experiment run the sample temperature was stabilized within $0.5^{\circ} \mathrm{C}$. The molecular axis orientation was fixed in such a way that it was either parallel or perpendicular to the direction of the electric field $\boldsymbol{E}$ (and simultaneously perpendicular to the wave vector $k$ ). The geometry of each case is presented in Fig. 2. Finally, we have reoriented the molecules in the sample so that their long axes were normal to its surface; in this case the vector $n$ was parallel to $k$. Such a configuration is invariant with respect to any rotation of the sample around the axis parallel to $k$ and consequently scattering from an ordered phase should then be similar to that in the isotropic liquid phase.

The thickness of the SLC-film was determined with the accuracy to $\lambda / 4$. The diameter of the incident beam was estimated to be about $2 \mathrm{~mm}$.

The outgoing beam was analysed by means of the Fabry-Perot (F-P) cavity, which was tuned in such a way that the pumping beam was entirely filtered out (reflected). In consequence, only waves with slightly different frequencies were transmitted. Hence the registered by us radiation was created in the process of 

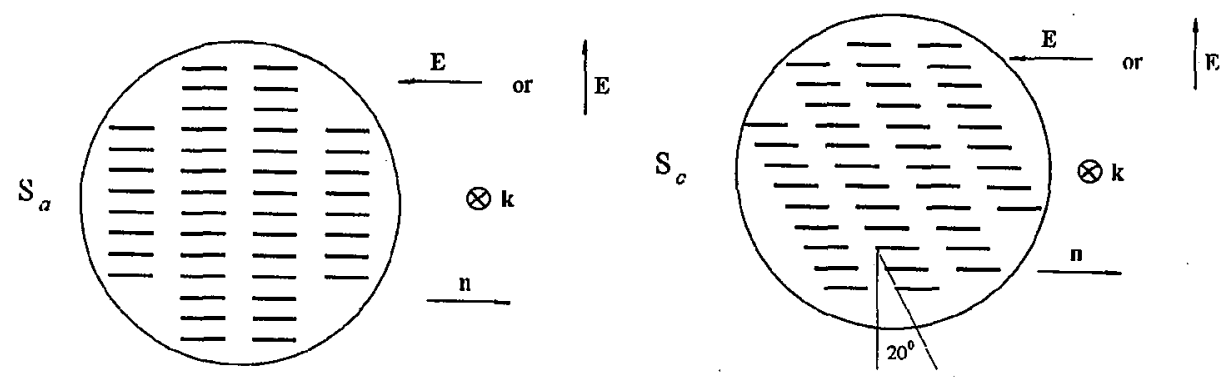

Fig. 2. Geometrical relations between the director $\boldsymbol{n}$ and the electric field vector $\boldsymbol{E}$ of the incident beam: (a) smectic phase $S_{a}$, (b) smectic phase $S_{c}$.

inelastic scattering. On the other hand, the beam reflected by the cavity passed again through the sample and was in part scattered backwards, i.e. in the direction of the original laser beam.

The accuracy of all intensity measurements was about $0.2 \mathrm{~mW}$.

\section{Results}

The results of measurements for two smectic phases $S_{a}$ and $S_{c}$ are presented in Figs. 3 and 4.

(a) For the smectic phase $S_{a}$ the maximum of scattered wave intensity appears for $\boldsymbol{E}$ parallel to the molecular long axes. The difference between the scattered intensities for $\boldsymbol{E}$ parallel or perpendicular to $n$ is not large, provided that the incoming intensity does not exceed the value closed to $300 \mathrm{~mW}$ (Fig. 3). Above

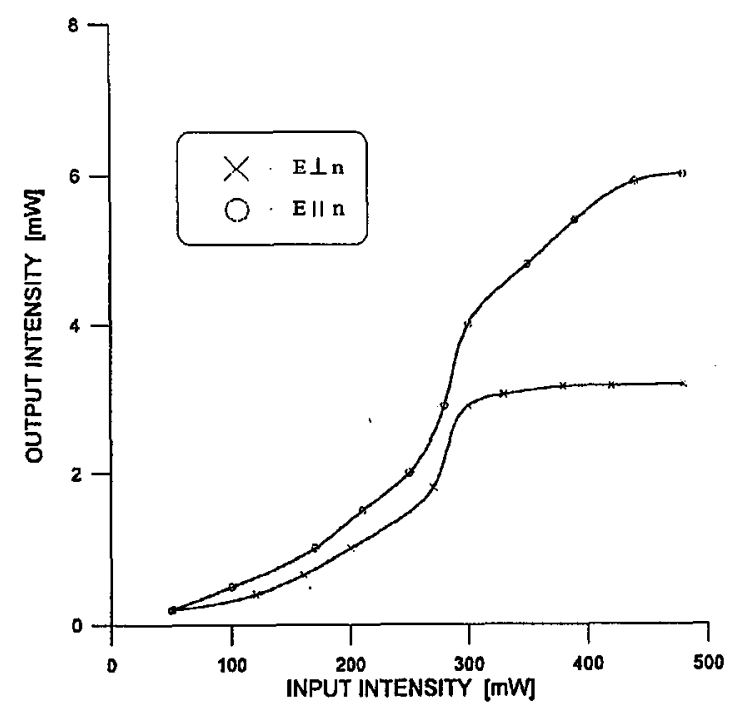

Fig. 3. Dependence of the transmitted light intensity on the incident light intensity for $S_{a}$ smectic for $\boldsymbol{E}$ parallel to $\boldsymbol{n}$ (upper curve) and $\boldsymbol{E}$ perpendicular to $\boldsymbol{n}$ (lower curve) at $t=45^{\circ} \mathrm{C}$. 


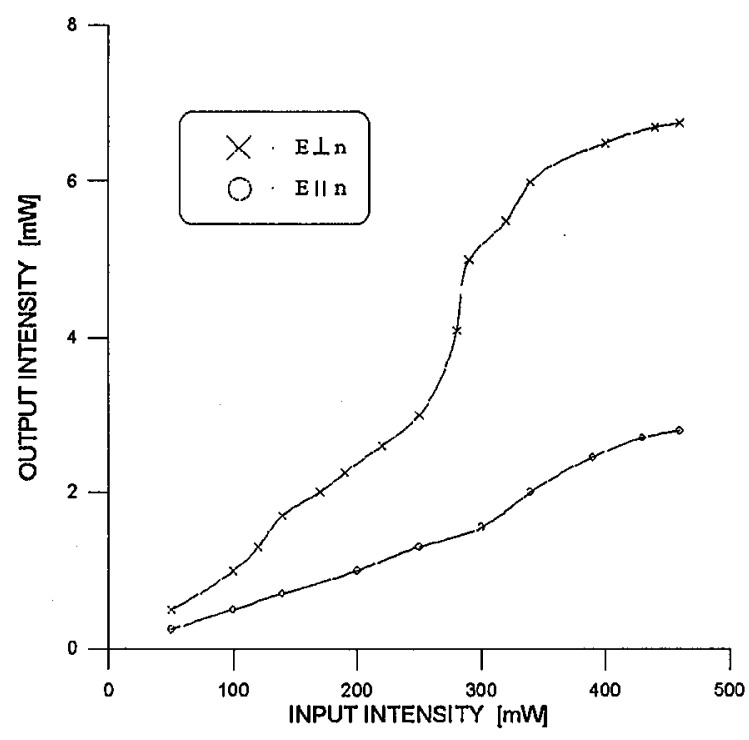

Fig. 4. Dependence of the transmitted light intensity on the incident light intensity for the phase $S_{c}$ for $\boldsymbol{E}$ perpendicular to $\boldsymbol{n}$ (upper curve) and $\boldsymbol{E}$ parallel to $\boldsymbol{n}$ (lower curve).

this value this difference becomes much larger for $\boldsymbol{E} \| \boldsymbol{n}$ than for $\boldsymbol{E} \perp n$. Both curves exhibit saturation and the upper limit is almost two times larger. In this region the scattering has a nonlinear character. We may thus conclude that nonlinear scattering strongly depends on the direction of the incident beam polarization with respect to molecular axes.

The above measurements were performed at a temperature of $45^{\circ} \mathrm{C}$. For other temperatures $\left(50^{\circ} \mathrm{C}\right.$ and $\left.55^{\circ} \mathrm{C}\right)$ similar results were obtained. On the other hand, when the temperature of the SLC sample was kept above the melting point (isotropic phase), no dependence on light polarization was observed. These facts suggest that a change in sample temperature is of secondary importance for the process of interaction of light with SLC, in the range of the temperature examined in our experiment.

(b) The role of the mutual orientation of vectors $\boldsymbol{E}$ and $n$ is reversed for light scattering in the smectic $S_{c}$ phase (Fig. 4). The molecular long axes are then twisted by the angle $20^{\circ}$ in comparison with the phase $S_{a}$. We have observed a larger scattered intensity when $n$ was roughly perpendicular to the electric vector $\boldsymbol{E}$ of the light beam. The maximal scattered intensity is then about two times larger than for parallel alignment of axes and $\boldsymbol{E}$.

\section{Physical background}

In liquid crystals the principal nonlinear optical effects are caused by a laser-induced director axis reorientation, as well as by temperature and density changes. Because of the highly anisotropic nature of liquid crystals in ordered 
phases, all the nonlinear effects strongly depend on the interaction geometry. A detailed analysis of these changes is given in a number of papers (see, for instance, Refs. [5-7]).

As mentioned earlier, the temperature for each experiment run was kept fixed with an accuracy of $0.5^{\circ} \mathrm{C}$. It therefore seems reasonable to assume that thermal effects played a secondary role in the scattering process.

The inelastic light scattering observed by us may have several different origins. The strong dependence of the scattering on relative directions of vectors $n$ and $\boldsymbol{E}$ suggests that a dominant contribution to the scattered intensity stems from processes involving a reorientation of molecular axes. The incident light beam always creates orientational waves as described in Ref. [2]. First, there appears a very weak electromagnetic wave with frequency $\omega^{\prime}$ and wave vector $k^{\prime}$. Its polarization vector $\boldsymbol{E}^{\prime}$ can in general be different from the vector $\boldsymbol{E}$ of the incident wave. Both waves interact nonlinearly, giving rise to an essential increase in the intensity of the secondary wave. The interference of these two waves leads to an orientational wave with frequency $\Omega=\omega^{\prime}-\omega$ and wave vector $q=k^{\prime}-k$. This wave is responsible for the Brillouin-type scattering. The maximal Brillouin scattering appears for the scattering angle $180^{\circ}$ [8]. In our experiments the scattered waves were observed in the forward direction. Therefore we have to assume that we measured scattering given by the wave reflected by the F-P cavity (see Fig. 1). The above interpretation suggests the stimulated character of the scattering process.

Another cause of nonlinear light scattering may be linked to Raman scattering. A maximum of Raman scattering corresponds to zero scattering angle and it may be generated by the original laser beam. It is, however, less sensitive to the molecular axes orientation than the Brillouin scattering and therefore seems to be of secondary importance in our measurements. The Brillouin scattering to which we refer above has a different nature from that encountered in other experiments, when acoustic waves induced by two interfering light beams are the main scattering centres. The existence of a periodic layered structure in smectic crystals strongly changes their elastic properties in comparison with those in nematics. A change of the layer period requires a large amount of work, to be performed by the incoming light beam. That is the reason why in the explanation of our experiment we neglected displacements of whole molecules which are the essence of acoustic waves.

There is another argument supporting our interpretation. The width of the frequency spectrum transmitted through the $\mathrm{F}-\mathrm{P}$ resonator is relatively very small (of the order of $10^{4} \mathrm{~Hz}$ ) so that the frequency of the transmitted wave lies close to the primary one. Such a picture corresponds to the Brillouin scattering (here: the stimulated scattering).

\section{Conclusions}

In this paper we have presented some preliminary experimental results concerning the influence of polarization vector and molecular orientation on the intensity of light scattered by a smectic liquid crystal. As a medium we used a particular type of smectic liquid crystal which can be ordered into several different 
orientational phases. Therefore it is a very suitable tool for investigation of light polarization effects.

We observed a strong dependence of the scattered intensity on the relative directions of polarization vector and molecular orientation. It appeared that this effect did not essentially depend on the temperature.

The question of the origin and mechanism of the scattering process is not entirely clear. We have proposed a possible mechanism of light interaction with the material. Our set of experimental data is still incomplete and further investigations are necessary.

\section{Acknowledgment}

This work was partially supported by the grant No. 8 S507 01806 of the Committee for Scientific Research,

\section{References}

[1] P.N. Prasad, Introduction to Nonlinear Optical Effects in Molecules and Polymers, John Wiley \& Sons, New York 1990.

[2] N.V. Tabiryan, A.V. Sukhov, B.Ya. Zeldovich, Mol. Cryst. Liq. Cryst. 136, 1 (1986):

[3] I.C. Khoo, Liquid Crystals: Physical Properties and Nonlinear Phenomena, John Wiley \& Sons, New York 1995.

[4] P.G. de Gennes, P. Prost, Physics of Liquid Crystals, Clarendon Press, Oxford 1995.

[5] G. Cipparone, C. Umetom, G. Arabia, G. Chidichimo, F. Simoni, Mol. Cryst. Liq. Cryst. 179, 87 (1991).

[6] I.C. Khoo, Mol. Cryst. Liq. Cryst. 207, 317 (1991).

[7] I.C. Khoo, G.M. Finn, J.Y. Hou, T.H. Liu, R.R. Michael, P.Y. Yan, J. Opt. Soc. $A m . B$ 4, 886 (1987).

[8] B. Berne, R. Pecora, Dynamic Light Scattering, Wiley-Interscience, New York 1976. 